\title{
Multi-Goal Optimization in PowerMatching City: A Smart Living Lab
}

\author{
Jan Pieter Wijbenga ${ }^{1}$, Pamela MacDougall ${ }^{1}$, Rene Kamphuis ${ }^{1}$, Tjerk Sanberg ${ }^{2}$, Albert van den Noort ${ }^{2}$, Elke Klaassen ${ }^{3}$ \\ ${ }^{1}$ TNO, Netherlands, ${ }^{2}$ DNV GL, Netherlands, ${ }^{3}$ Eindhoven University of Technology, Netherlands \\ jpwijbenga@tno.nlＴjerk.Sanberg@dnvgl.comＥ.A.M.Klaassen@tue.nl
}

\begin{abstract}
Whereas other smart grid field trials optimize for a single objective, PowerMatching City phase 2 demonstrates that multiple objectives can be achieved in a coordinated virtual power plant. A system that incorporates both consumer community proposition incentives and trade dispatch incentives (as well as end-user comfort) was designed, implemented and tested. This paper describes the design approach and the main findings in this real life field trial. The multi-goal approach showed to be successful, as the individual goals were achievable by the cluster. To prevent a single goal from overpowering the other objective limitations and a flexibility-balancing component were implemented. Finding the optimal mix of balancing the components and utilizing the most of the flexibility requires additional research.
\end{abstract}

Index Terms - Virtual Power Plant, Smart Grid, Field Trial, Demand Response, ICT, Renewable Energy, Multi-Agent Systems.

\section{INTRODUCTION}

Whereas similar smart grid field trials like EcoGrid [8] and Couperus [7] optimize for a single goal, PowerMatching City phase 2 tries to solve multi-objective optimization in a single cluster.

PowerMatching City is the first living lab smart grid in Europe [1]. It's located in Groningen, in the Northeast of the Netherlands. In a successor project, appliances in 40 households are being coordinated within the smart grid. The distributed coordination technology, called PowerMatcher, is a multi-agent-based system that uses electronic exchange markets to coordinate a cluster of devices to match the electricity supply and demand. All appliances are represented by an agent, that is entrusted with the optimization of the device's objective. Every agent defines a bid that represents the allocated power for a given PowerMatcher price range. To avoid confusion, from now on the PowerMatcher price will be called the total incentive throughout this paper. The auctioneer agent aggregates the bids and finds the point where supply and demand meet. This is the total incentive and it is sent down to the agents, who determine their allocated power by finding this total incentive on their most recent bid. Agents are not restricted to appliances; also energy retailers may have a socalled objective agent that produces bids to achieve their goals. The operation of the PowerMatcher is summarized in Fig. 1. More detailed information on the PowerMatcher technology and associated experiments can be found in [2].

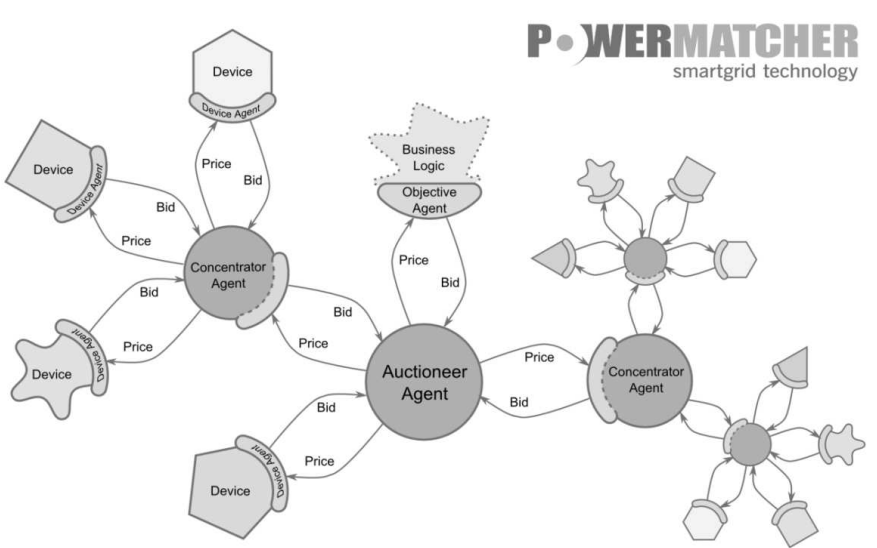

Figure 1. Schematic overview of the PowerMatcher concept [2]. Note that in this field trial the term total incentive is used, rather than price, since it is a steering signal and it incorporates different goals.

This paper discusses the results of the phase 2 extended field test regarding multi-objective optimization. It is shown that the individual goals are achieved through incentives, a self-balancing system was created and that the total or combined incentive correlates with the actual measured power of the flexible devices.

The rest of this paper is structured as follows. Chapter 2 describes the project goals. The PowerMatching City setup is described in chapter 3. Chapter 4 discusses the implementation of services. Chapter 5 lists the main results. The final chapter features the discussion and conclusion and it offers suggestions for future research.

\section{PROJeCt GoALS}

PowerMatching City spawned from the European project INTEGRAL [4] as one of three demonstrations, each focusing on solutions for "Emergency", "Critical" and "Normal" grid operation. PowerMatching City demonstrated new smart grid solutions for "Normal" operation. Its main goal was to develop and demonstrate a combination of three types of coordination of distributed energy resources for technical (congestion management), commercial (energy trading) and in-home optimization. These different coordination types are graphically displayed in Fig. 2. The figure also shows that different households may choose any commercial aggregator and that they may be connected to different parts of the network. 


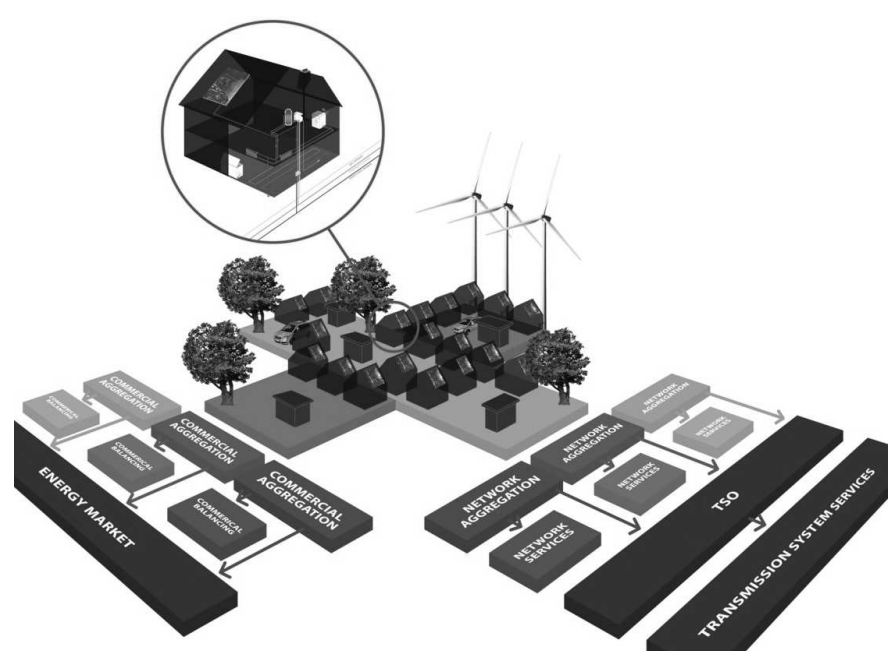

Figure 2. Overview of stakeholders in PowerMatching City, coordination is based on technical, commercial and in-home optimization.

In PowerMatching City simultaneous optimization of the system was achieved using a multi-layered PowerMatcher network. Use cases with multi-goal optimization, addressing the interests of three stakeholders simultaneously, were investigated, involving the consumer, distribution system operator and the commercial aggregator (energy trader). The use case studied specifically investigated the feasibility of local peak load reduction, imbalance reduction as well as the most cost effective use of energy in-home [3]. Phase 2 of PowerMatching City saw the technical feasibility study expanded with innovative energy services, in order to investigate consumer preferences within the smart living lab. Therefore, two types of end-user propositions were created: Smart Cost Saving and the Sustainable Community proposition. Furthermore, the second phase of PowerMatching City incorporates forecasting for balancing services in the trade dispatch optimization and a revised control mechanism of the distribution system operator.

The Smart Cost Saving proposition focuses on the scenario where customers of a community are being charged according to a dynamic tariff based on the trade portfolio position on the day-ahead market. As a result their flexible devices are optimized to offer demand response at the most cost effective times of the day. The Sustainable Community proposition incentivizes customers to consume when there is locally generated renewable energy and to postpone consumption when sufficient local energy is expected in the near future. This proposition takes into account both the forecast of the individual household photovoltaics as well as the community solar generated electricity forecast.

\section{PowerMatching City SETUP}

The backbone of PowerMatching City is 40 standard households, located in and around the city of Groningen, the Netherlands. Each are fitted with either a domestic combined heat and power unit (micro-CHP) or a hybrid heat pump system with gas fired heater and around $14 \mathrm{~m}^{2}$ of photovoltaic (PV) panels. Some households also contain an intelligent washing machine. All devices are interfaced with PowerMatcher software agents to operate PowerMatching City as a virtual power plant (VPP).

The 23 air-water heat pumps are used for base load heating throughout the season, while the gas fired heaters provide additional heat during peak heat demands and for domestic hot water. The 17 micro-CHPs have an electric capacity of $1 \mathrm{~kW}$ and provide heat for both base and peak load heating. The micro-CHPs and heat pumps are connected each to a 230 litre thermal storage tank, which allows the heating devices to be turned on or off independently of the immediate heat demand of the household, thus providing flexibility to the smart grid [5].

The VPP resulting from the cluster of households was steered using a trade dispatch objective software agent (TDOA) strategy. This agent was developed to allow real-time response in the market taking into account prior optimization of demand response and power generation in view of the expected external day-ahead market electricity price as well as operational adjustments needed in view of the real-time position of the portfolio on external markets [6]. This will be further discussed in the next section. Each household was assigned randomly to one of the two propositions, Sustainable Community and Smart Cost Saving, to allow concurrent evaluation of both propositions. The 20 households in the Sustainability Community proposition comprise 12 heat pumps and 8 microCHPs, while the 20 households in the Smart Cost Saving proposition contain 10 heat pumps and 9 micro-CHPs. The proposition method enabled the households to not only respond to trade dispatch objectives of a commercial aggregator, but also to that of the personal consumer preferences. Apart from these two goals, there is the objective of the distribution system operator to reduce peak loads. In the course of the project, this objective will be incorporated in the system as well, based on a revised control mechanism. This paper shows the results of simultaneous optimization of consumer preferences and trade dispatch without peak load management.

\section{IMPLEMENTATION OF SERVICES}

At the top level of the VPP is the TDOA [6]. The TDOA uses the price profile of the day-ahead spot market to optimize the energy profile of the cluster. The total power and flexibility is forecasted and an optimization algorithm constructs an optimal forecast for the next day. Additionally, the TDOA can offer regulatory power to the national system operator for balancing purposes in real time. In this case there will be a deviation from the day-ahead energy profile in order to provide regulatory power that is requested by the system operator, in order to reduce system imbalance. The result of these optimization steps is fed into the PowerMatcher system as a flexibility offer. The bid of the objective agent will make the PowerMatcher auctioneer alter the total incentive for all the agents. The size of the flexibility offer by the TDOA was unconstrained at first, but after a phenomenon called incentive clipping occurred the size of the bid was constrained so that the 
TDOA did not overpower the other goals. Fig. 3. shows an overview of the TDOA optimization strategy.

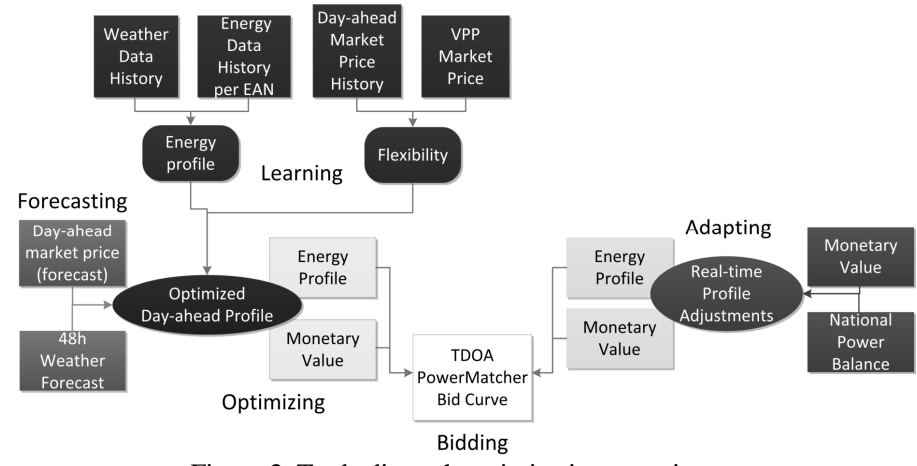

Figure 3. Trade dispatch optimization overview.

The second objective of VPP optimization is the consumer propositions. In interactive co-creation sessions, consumers designed two distinct consumer propositions based on common personal preferences. One proposition focused on lower electricity bills and the other proposition entailed using sustainable, locally generated energy within the community. In the field trial, each household was assigned a preference for energy optimization, either on Sustainable Community or Smart Cost Saving. Without these propositions the households would offer their complete flexibility in their PowerMatcher bids to the VPP, i.e. the amount of flexibility in the thermal storage or the time until a wash cycle must be completed.

To what extent the propositions influence the load is configurable. Both propositions give incentives to bring forward or postpone energy demand. Consequently this incentive is incorporated in the PowerMatcher flexibility offer in order to influence the market equilibrium. The architecture of the integration of propositions in the PowerMatcher is shown in Fig. 4.

The Smart Cost Saving proposition uses the incentive to reduce the households' electricity bills. The assumption behind the proposition is that consumers are charged an hourly tariff for their electricity consumption. This hourly tariff is assumed to be known beforehand. The proposition optimization looks at the price now and the price in an hour and decides if it is best to postpone electricity demand or to stimulate it now. In this way, flexibility is used to shift load away from time periods with a high market price. The input for the proposition optimization is considered an additional service by the energy supplier.

The Sustainable Community consumers are incentivized by consuming locally generated, renewable energy. Therefore, their flexibility offers are optimized in such a way that when there is a large amount of PV generation the offer is steered more towards consuming electricity. This is based on the PV generation of individual households and if the household's PV is not above the configured threshold the total community generation is considered, so that households with little or no PV power can use the PV power of their fellow community members.
It should be noted that a maximum of 20 percent of the available flexibility is configured to be available to steer towards personal preferences, the remainder of the flexibility range is utilized for the multi-goal TDOA optimizations.

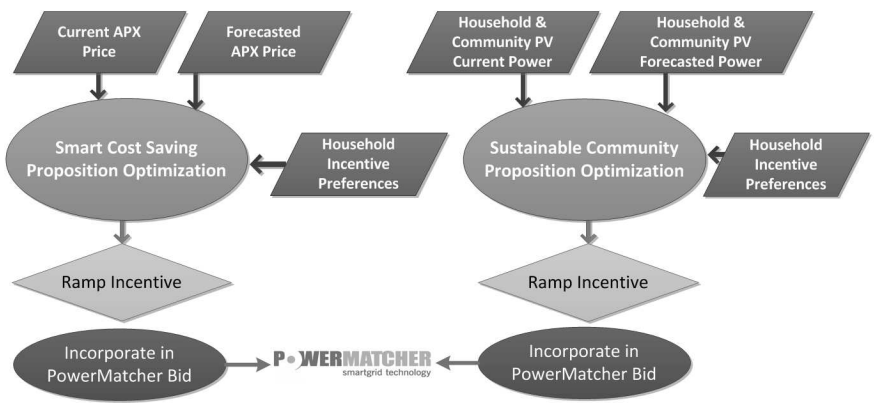

Figure 4. Integration of propositions in the PowerMatcher architecture.

\section{EXPERIMENTAL RESULTS}

The Sustainable Community proposition acts on a single day, shifting energy consumption and production based on the presence or the expectation of PV generation. A positive incentive is sent by the proposition based on the current absence but expected future generation. Positive incentives postpone electricity consumption, e.g. the load of heat pumps, and bring forward electricity production, e.g. the generation of micro-CHPs. Once the PV panels start producing, the incentive becomes negative and opposite behaviour is encouraged. An example of a Sustainable Community proposition incentive for one single day is shown in Fig. 5.

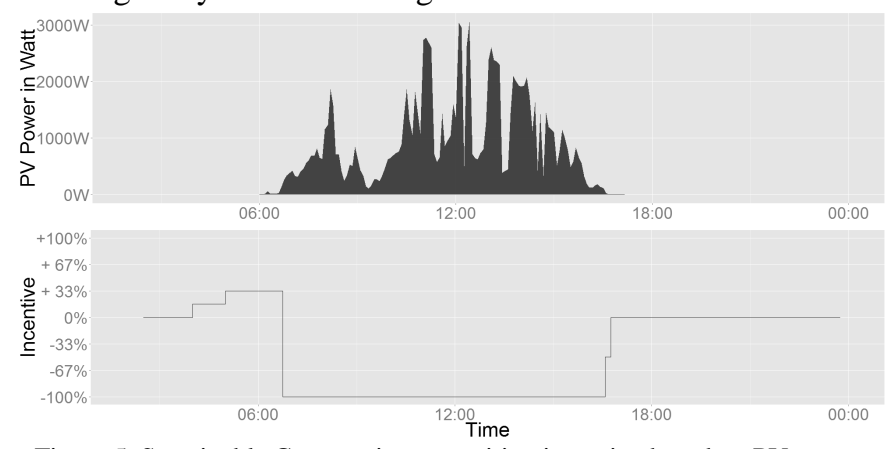

Figure 5. Sustainable Community proposition incentive based on $\mathrm{PV}$ power.

As an example of this, results from a single heat pump can be seen below. The behaviour of one heat pump follows from a combination of the incentive, user comfort setting and the other optimization goals. Around 6:00 the warm water buffer is filled to guarantee user comfort using the auxiliary gas-fired burner. This is due to the heat pump's limitation that it is not working when the outside temperature is below 5 degrees Celsius, occurring in the early morning. Later in the day, the heating by electrical means occurs simultaneous with the peak in solar power and enforces a higher buffer level. 


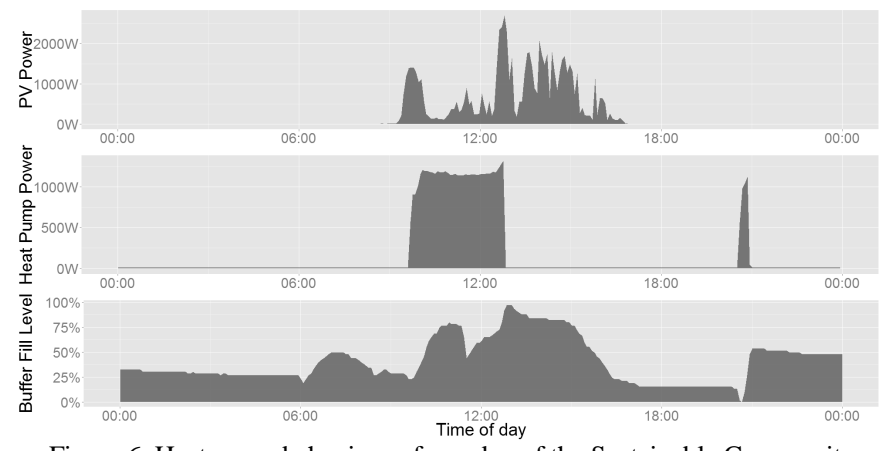

Figure 6. Heat pump behaviour of one day of the Sustainable Community proposition.

These results show that on an individual level the households were influenced by the desired behaviour of the proposition. Analysis of the sustainable community as a whole showed a correlation between the available PV power and the combined heat pump and CHP power. When PV power is high the heat pumps are more likely to consume electricity and when the PV power is low, the CHPs are more likely to produce electricity.

Consumers in the Smart Cost Saving proposition their production and consumption based on what they will be charged. The proposition implementation looks ahead one hour and if the price increases it will stimulate immediate electricity consumption and delay electricity production by means of a negative incentive. When prices are expected to drop, devices are steered towards delaying consumption. Figure 7 shows that the incentive is implemented to react to price increases or decreases above a certain absolute threshold.

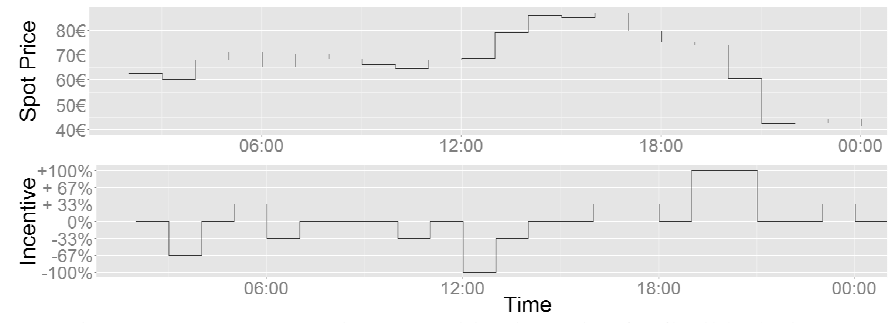

Figure 7. Smart Cost Saving proposition incentive for flexible devices.

The incentives of the proposition directly influence the total incentive. The next point of proof is to show that the total incentive actually correlates with the measured power values of the flexible devices in the cluster. This is shown in Fig. 8, where the average heat pump load during each timeslot (5 min. time resolution) is plotted against the total incentive. The correlation based on linear regression between the load and the total incentive is significant $(P$ value $=0)$, the correlation coefficient is $-2 \mathrm{~W}$, which means that the heat pump load is likely to increase by $2 \mathrm{~W}$ if the total incentive increases by $1 \%$. Additional research is required to investigate to what extent this relationship is influenced by external variables, such as: hour of day or weather circumstances.

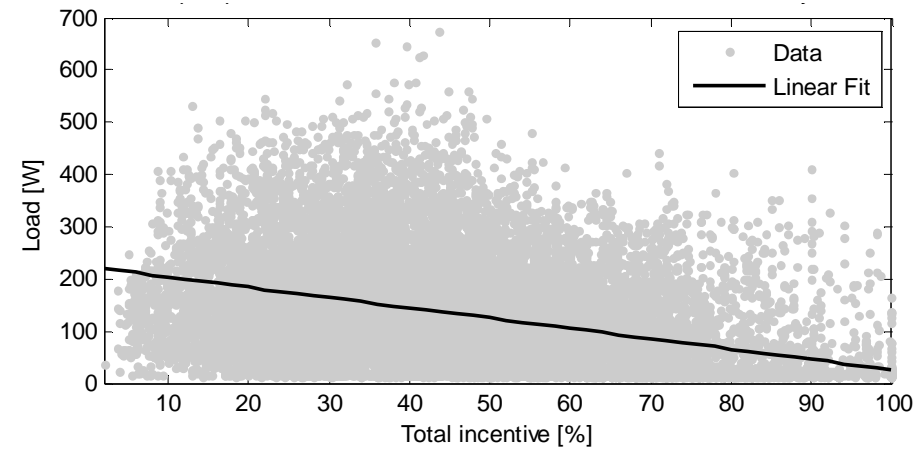

Figure 8. Correlation between heat pump load and incentive, based on data from PowerMatching City, from 01/03/2014 till 01/05/2014.

In addition to the proposition objectives, the TDOA objective of optimization for day-ahead spot market and imbalance showed to be successfully achieved by the cluster. This is shown in Fig. 9. that displays one day of VPP operation. It shows the aggregated heating system power in the VPP and the price profile that was used for optimization.

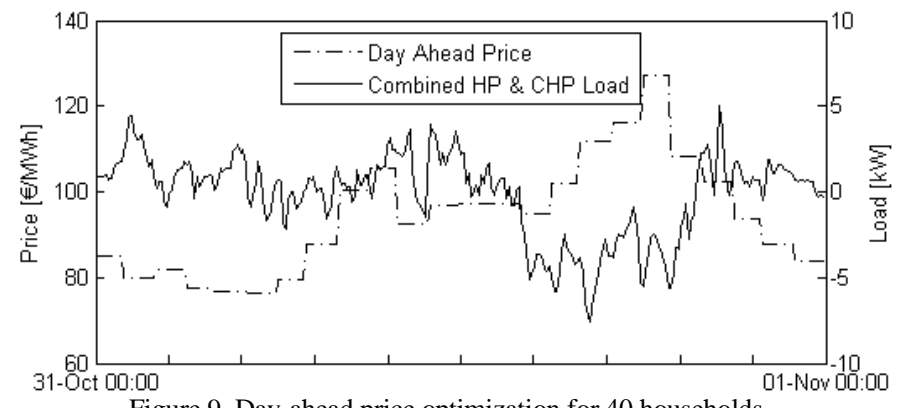

Figure 9. Day-ahead price optimization for 40 households.

During the period from March 1 till May 1 the TDOA successfully made and enforced an optimized forecast for its day-ahead portfolio. The day-ahead forecasted load and the actual realized load by the VPP can be seen in Fig. 10 .

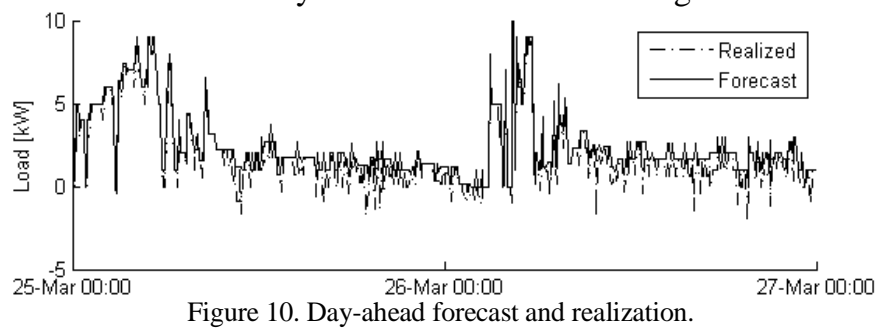

Initially the TDOA was implemented so powerful that the TDOA goal overpowered the other goals and struck the upper power limit of the flexibility of the VPP. When this happened a phenomenon called incentive clipping took place. This is the situation where the cluster equilibrium stays at the maximum or minimum incentive for a longer period. When this happens, further increase or decrease of the incentive is not possible even if it may be desired. In other words there is no flexibility in the VPP in one direction, ramp-up or ramp-down power is not achievable.

To counteract this effect two measures were taken: limitation of the TDOA and the introduction of a balancing 
component. Limitation of the TDOA was achieved by constraining the size of the flexibility offer of the TDOA. The balancing component was incorporated into the TDOA. Whenever the incentive would approach the upper or lower boundary the TDOA will alter its control for a period of time to restore the cluster to a more balanced state, where both rampup and ramp-down is possible again for the VPP. This short period of balancing can be seen as recovery time for the cluster's flexibility potential, similar to the situation where a household with a full hot water buffer cannot store more energy, but subsequent domestic heat demand drains the heat pump buffer, so that the buffer may be charged intelligently later on.

The period of incentive clipping and the period of subsequent stability are shown in Fig. 11. It shows the development of the total PowerMatcher incentive in the cluster: the overall steering signal that is obtained from matching the bids of all the households. The configured allowed range is between 1 and 100. When the incentive reaches 100 electricity consumption is delayed maximally and production is promoted maximally. From March 1 to May 1 (and after that) the PowerMatcher-based incentive system is able to coordinate supply and demand in the cluster utilizing the flexibility in the buffers. Also it may be concluded from this graph that there is some unutilized flexibility, because the minimum and maximum of the total incentive are not often reached. Increasing the power of the objectives by means of the built-in configuration parameters will lead to a more optimal solution.

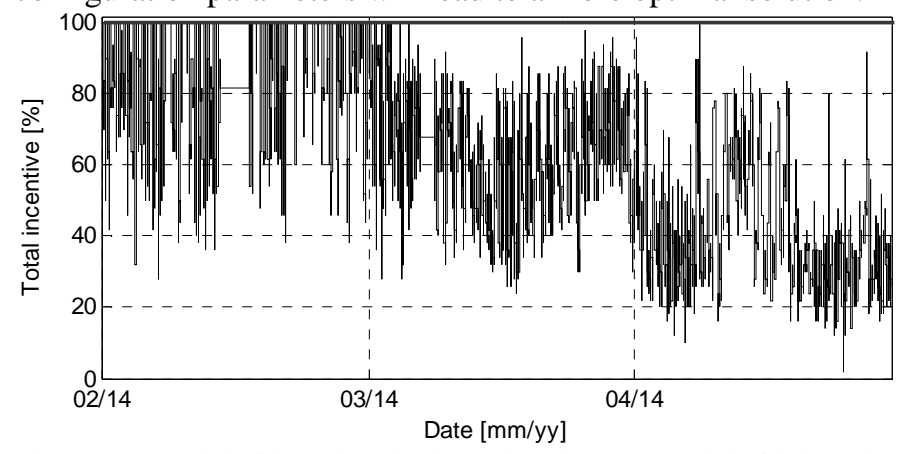

Figure 11. A period of incentive clipping and a subsequent period of balance in the cluster.

\section{DISCUSSION \& CONCLUSION}

Initial results of PowerMatching City phase 2 show that this real-life VPP is able to handle balanced community propositions in addition to the goals and optimizations of a trade dispatcher. The results presented here show a positive outcome, however further research into the design and configuration of such a VPP is required. Further research could consider how the task of managing the flexibility level in the cluster can be implemented optimally. Furthermore, the current approach entitles the different goals to influence the cluster continuously and simultaneously. Future research may investigate different approaches for distributing flexibility, e.g. the approach where one stakeholder per timeslot may have the single right to use the total flexibility for a fixed period of time (and afterwards restores the balance in the VPP for the next candidate). Overall, it was shown that it is possible to individually incentivize customers while maintaining the trade goals of the energy retailers while retaining a balance in this real-life virtual power plant.

\section{ACKNOWLEDGEMENTS}

The authors thankfully acknowledge all of the participants of PowerMatching City for allowing the consortium to explore the energy system of the future in their houses. The consortium partners Essent, Enexis, Gasunie, ICT Automatisering, TNO, DNV GL and the IPIN subsidy program of the Dutch government are greatly acknowledged for making this project possible.

\section{REFERENCES}

[1] F.W. Bliek, A. van den Noort, B. Roossien, I. G. Kamphuis, J. de Wit, J. van der Velde, and M. Eijgelaar, "PowerMatching City, a living lab smart grid demonstration," in Proc. IEEE PES Innovative Smart Grid Technologies Conf. Europe, 2010.

[2] K. Kok, "The PowerMatcher, Smart Coordination for the Smart Electricity Grid". PhD thesis, Vrije Universiteit Amsterdam, 2013.

[3] K. Kok, B. Roossien, P.A. MacDougall, O.P. Pruissen, G. Venekamp, I.G. Kamphuis, J.A.W. Laarakkers, and C.J. Warmer, "Dynamic pricing by scalable energy management systems - field experiences and simulation results using PowerMatcher". In Proc. IEEE Power and Energy Society General Meeting, 2012.

[4] FP7 Project Integral Website. http://www.integral-eu.com. 2014.

[5] F.W. Bliek, A. van den Noort, B. Roossien, I.G. Kamphuis, J. de Wit, J. van der Velde, M. Eijgelaar, "The role of natural gas in smart grids". In Proc. International Gas Research Conference, 2011.

[6] I.G. Kamphuis, B. Roossien M. Eijgelaar, H. de Heer, J. van de Velde, A. van den Noort, "Real-time trade dispatch of a commercial VPP with residential customers in the PowerMatching City SmartGrid living lab". In Proc. International Conference on Electricity Distribution, 2013.

[7] Couperus Smart Grid: Proeftuinen Intelligente Netten, "http://www.rvo.nl/sites/default/files/2013/09/Couperus\%20Sm art\%20Grid_0.pdf”, July 2013.

[8] EcoGrid EU: From Design to Implementation, "http://www.euecogrid.net/images/News/131004_\%20edk\%20a4_ecogrid\%20e u\%20project_web.pdf", 2014. 